\title{
SHAPE CONTEXT BASED CLUTTER DEFIANCE IN LOGARITHMIC CORRELATION FILTERS
}

\author{
Ahmed Bilal Awan ${ }^{1}$, Asim D. Bakhshi², Farhan Riaz ${ }^{1}$ and Saad Rehman ${ }^{1}$ \\ Department of Computer Engineering, EME College, \\ ${ }^{I}$ National University of Sciences and Technology (NUST), Islamabad, Pakistan \\ ${ }^{2}$ Department of Computer Engineering, Abasyn University, Islamabad, Pakistan
}

\begin{abstract}
Correlation based classification decisions are challenged by the occurrence of clutter in the real life input scenes. Clutter tends to cause side-lobes in the output correlation plane thus making correlation based classification decision nontrivial. The pronounced side-lobes may dominate correlation peak for true class objects leading to a false alarm. We propose a new hybrid scheme involving shape context and correlation filters to avoid impact of clutter in correlation output. The proposed combination provides a dual validation strategy where presence of true class object in test image is confirmed at two different stages before final class decision. Clutter participation in correlation process is denied by matching shape contexts of each object in test image with the true class object. The recommended addition in optimal logarithmic maximum average correlation height filter $(\mathrm{LMACH})$ results in a performance gain ranging from $30 \%$ to $70 \%$ in peak to side-lobe ratio leading to a correct classification decision. The improvement remains consistent throughout the range of in-plane angular distortions against synthetically cluttered challenging images.
\end{abstract}

\section{KEYWORDS}

Correlation Filters, Object Recognition, Correlation Pattern Recognition (CPR), MACH, LMACH, Shape Context

\section{INTRODUCTION}

Although being a basic mathematical operation, correlation function has become an important component of computer vision techniques to resolve object recognition problem. A comprehensive machine vision capability still remains a challenge thus needing an appropriate pre-processing coupled with correlation operation to answer some essential questions. A number of such algorithms have been devised in the past resulting in various brands of correlation filters that show a promising recognition performance against relatively complex input scenes (Vijaya Kumar et al. 2005, Mahalanobis et al. 1994 \& Alkanhal et al. 2000). Further optimization of correlation filters is achieved by inculcating logarithmic translation in the design (Ho et al. 2002), thereby accumulating rotation (both in-plane and out-of -plane) and scale invariance capability in a single solution (Bone et al. 2006). However, the task of recognition becomes significantly complex in real time due to the frequent occurrence of clutter alongside presence of background. Recently, a remarkable technical advancement has been viewed in the recognition domain while employing feature based methods. One of the most convincing methods involved shape contexts as descriptors for the object identification.

The logarithmic correlation filters being a composite package for distortion invariance is challenged by the occurrence of clutter and backgrounds that indirectly tend to impact classification decisions by distorting correlation output through occurrence of side lobes (Vijaya Kumar et al. 2005). Whereas, the feature based methods can supplement correlation filters to evade clutter while maintaining distortion invariance. This study is focused on utilizing strengths of techniques based upon correlation filters and feature based classifiers to augment each other.

The proposed hybrid scheme titled as "Shape Context based Logarithmic Maximum Average Correlation Height Filter" (SC-MACH) uses shape contexts by Belongie et al. (2002) as features to ascertain the presence of target object in the input scene with subsequent validation by the correlation filter. The presence of target is determined on the basis of shape contexts specific to that particular object. This process also entails a 
mechanism of discarding false objects/ clutter during the course of ascertainment of true object. Resultantly, a clean test image is reconstructed before carrying out correlation with the reference image.

We evaluate logarithmic Correlation filter design with a focus on optimal MACH filter in Section 2. The next section covers an overview of shape context alongside histogram matching and optimal cost assignment. Adopted methodology is discussed in section 4, followed by the performance evaluation, results and discussion. Some conclusive remarks are given in the end.

\section{LOGARITHMIC CPR FILTERS}

CPR adopts a statistical pattern recognition technique to generate a reference image that enables machines to identify presence of the target object in input image by observing the level of matching with the reference image. The degree of resemblance observed in output correlation-plane, is used to classify images. A variety of filter designs have been discovered so far with some common goals such as distortion invariance, noise tolerance and clutter rejection (Vijaya Kumar et al. 2005). A basic optimal MACH filter in frequency domain (Hanying and Tien-Hsin 1999) can be represented as following:-

$$
H=\frac{M^{*}}{\alpha C+\beta D_{x}+\gamma S_{x}}
$$

Where $\mathrm{M}$ is average of all training images $\left(X_{1}, X_{2}, \ldots \ldots, X_{N}\right)$ in frequency domain, and $\alpha, \beta$ and $\gamma$ are non-negative parameters. The diagonal power spectral density matrix of additive input noise represented by white noise covariance matrix $C=\sigma^{2}$ I. $D_{x}$ is the diagonal average power spectral density of the training images, which can be represented as:-

$$
D_{x}=\frac{1}{N} \sum_{i+1}^{N} X_{i} X_{i}^{*}
$$

Where, $X_{i}$ corresponds to the $\mathrm{i}^{\text {th }}$ training image in frequency domain, $N$ represents total number of training images, and $*$ depicts conjugate. $S_{X}$ stands for the similarity matrix of the training images:

$$
S_{x}=\frac{1}{N} \sum_{i=1}^{N}\left(X_{i}-M\right)^{*}\left(X_{i}-M\right)
$$

Varying the image representation from 2D Cartesian plane to a logarithmic plane allows us to view the angular rotation and scale alterations in the target object as equivalent shifts in memory (Ho et al. 2002), which are better understood by the machines. CPR filters when coupled with logarithmic conversions, yield some remarkable advantages. The blend of techniques has been profitably undertaken in the past (Bone et al. 2006 and Rehman et al. 2013), while joining log-polar mapping with different versions of CPR filters known as correlation filters. These filters with an inherent invariance to the out-of-plane rotations are augmented by the logarithmic mapping which caters for the complete range of in-plane rotations alongside scale changes (ranging between 0.5-2.5 times object size) and illumination effects. Although these filters have a good response against occlusions but presence of clutter distorts the recognition performance of log-CPR filters.

\section{SHAPE CONTEXT BASED MATCHING}

Object matching based on the shape context explores the similarity measure by correspondence between points on the two shapes. These correspondences are used to estimate alignment cost of two objects. Interest points are selected on each object. The distribution of all other points on the same object to the reference point is unique for each shape and this context can be effectively used as a descriptor of a specific shape. Shape context of two objects having similar shapes is identical.

For each point on object-1 we calculate shape contexts in the form of distribution of vectors joining $\mathrm{n}^{\text {th }}$ point with all other $\mathrm{n}-1$ points on the same object. This distribution of vectors for each point at object- 1 is translated into histogram having log-polar bins. Consequently we get $\mathrm{n}$ number of histograms for $\mathrm{n}$ interest points on object-1 and n' histograms for n' number of points at object-2 (where $\mathrm{n}$ and $\mathrm{n}$ ' can or cannot be equal). The histogram cost matrix is obtained by computing pair wise cost between all shape contexts represented by log polar histogram arrays of reference and test images. 


$$
C_{i j}=C\left(p_{i}, q_{j}\right)=\frac{1}{2} \sum_{k}^{K} \frac{\left[h_{i}(k)-h_{j}(k)\right]^{2}}{\left[h_{i}(k)+h_{j}(k)\right]}
$$

Where $h_{i}(k)$ and $h_{j}(k)$ are k-bin histograms (normalized by mean distance) for a point $\mathrm{p}_{\mathrm{i}}$ on object-1 and $\mathrm{q}_{\mathrm{j}}$ on object-2. Optimal cost assignment using Hungarian algorithm (applying row and column reductions) yields cost vector carrying coordinates of best matching points (Belongie et al.2002).

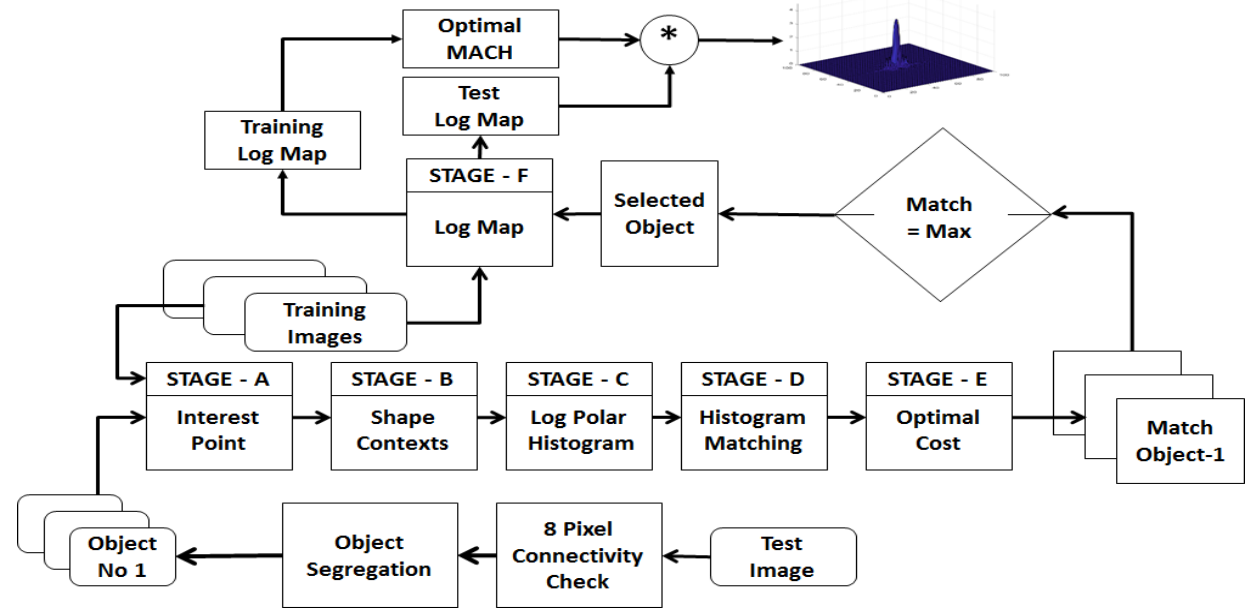

Figure 1. Block Diagram - Proposed Methodology

\section{METHODOLOGY}

We recommend image set having out-of-plane rotated versions of the target object shown in Figure 2a for training purpose. As in-plane rotation and scale invariance is inherently achieved by logarithmic mapping, training of correlation filter with out-of-plane rotated examples of target caters for other angular distortions.

Test image set includes synthetically cluttered images with added distortions as shown in Figure $2 b$. Object segregation in test image is performed through 8-pixel connectivity check before subsequent processing. The separated objects are independently passed through the stages A to E to learn level of matching of each object with the target object. As shown in block diagram (Figure 1), all training and test images are processed through stage A to stage $\mathrm{E}$ leading to the selection of test image containing target object. The log map of selected object (generated at stage F) are subsequently correlated with reference image (generated by LMACH) to validate results. Processing at each stage is elaborated below.

Stage-A: Segregated objects in test image alongside training images are input to this stage. Interest points are calculated for each object in the image. Sampled points on edges describe the presence of each object in the image. Coordinates of each interest point of segregated objects in test image and target objects in training images are output of this stage. As training images contain out-of-plane rotated views of target object, interest points that tend to exist in multiple views are identified. These common interest points accumulate to represent training data. Whereas, test data set is sequentially built by recording the set of interest points separately for each object in test image.

Stage-B: Training and test data in the form of coordinates of interest points is input to this stage. Each interest point in a single combined training image and multiple test objects participate to generate independent shape contexts respectively. The set of distance vectors originating from each interest point in an image to all other points within the same image form shape context as a specific representation of the object present in that image. As output of stage $\mathrm{B}$, training and test data set is translated into the shape context information.

Stage-C: Shape context information is further transformed into logarithmic polar histograms at Stage C using 5 bins for $\log r$ and 12 bins for $\theta$. This provides us 60 bins representation against each interest point where descriptors for both training and test data are more sensitive to the nearby interest points than the farther points. 
Stage-D: All descriptors in training data are matched with the descriptors in test data pertaining to all separated objects in test image using (Equation 8). Histogram cost matrices for all separated objects in test image are obtained, where each element in a matrix represents matching cost of a training interest point with a specific test interest point.

Stage-E: This stage involves solving optimal cost assignment problem using Hungarian algorithm (Belongie et al. 2002). Cost matrices are squared by adding dummy elements before carrying out cost assignment. For each cost matrix pertaining to a specific test object, the best matching interest points are identified with the points in training data based upon lowest matching cost. The points matching to the dummy elements are discarded and only good matches against each test object are recorded. Information regarding number of good matches is observed against each segregated test object and the one having maximum number of good matches is picked up as a selected object in the test image. The remaining objects in test image with lower number of matches are discarded while considering these objects as clutter.

Stage-F: After the relevant test object selection based on matching shape context, the selected test object alongside a complete set of training images are input to logarithmic polar transformation. Logarithmic polar maps for the test and training images are separately obtained. The training logarithmic maps are used to carry out training of MACH filter. Consequent to the output of correlation between MACH filter and logarithmic map of the selected test object, we can undertake the final detection decision.

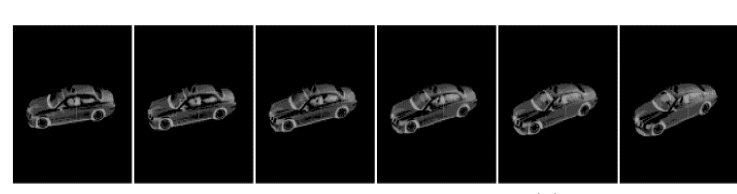

(a)

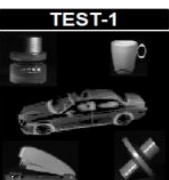

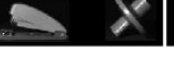

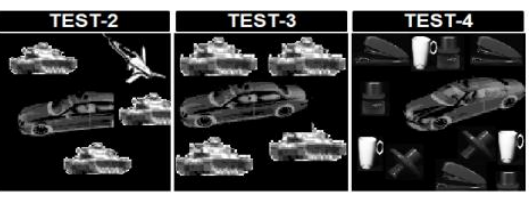

(b)

Figure 2. Data Set, (a) Training Images $\left(0^{\circ}-25^{\circ}\right.$ out-of-plane rotated versions of a car), (b) Synthetically cluttered Test Images with added complexity level

\section{PERFORMANCE EVALUATION}

The training set (shown in Figure 2a) contains out-of-plane rotated images each having $5^{\circ}$ angular differences $\left(0^{\circ}-30^{\circ}\right)$. Whereas, synthetically cluttered test images with multiple distortions (shown in Figure $2 \mathrm{~b}$ ) were used for performance evaluation of the proposed design. To evaluate for the whole angular distortion range, test images were exposed to in-plane rotations ranging from $0-360^{\circ}$ with $45^{\circ}$ separation. Rotated versions of Test-2 are shown in Figure 3a. Objects were segregated based upon 8-pixel connectivity in each in-plane rotated test image. The separated objects in the example test image are displayed in Figure $3 \mathrm{~b}$. Each of the segregated objects was passed through Stage A to C for generation of test data in the form of 60 bin log-polar histograms accrued from shape context information of that object. On the parallel, training images were consolidated as a single training image based upon accumulated common interest points after Stage A and the same was passed through Stage B and C for obtaining training data.

Histogram matching between test data pertaining to the segregated object and training data of consolidated training image was carried out at stage D. Subsequently, Hungarian optimal cost assignment algorithm was applied on cost matrices (against each object in Figure 3b) to determine the perfect match of each interest point. Based upon maximum number of matching interest points, the valid test object was selected out of test image and remaining objects were discarded. Figure $3 \mathrm{c}$ displays the selected object for the example test image, where other non-target objects/ clutter are wiped off. All training images and selected test object were logarithmically transformed to produce training and test logarithmic maps. The reference image was constructed by applying optimal MACH on training logarithmic maps (referred as Optimal LMACH in Figure 1). Logarithmic map of the selected object in test image was correlated with the reference image to validate detection of target object.

The correlation output peak intensity (COPI) corresponding to the selected image was compared to a pre-selected threshold value and occurrence of the target object was registered. The performance of CPR filters is benchmarked by a conventional parameter COPI (Vijaya Kumar et al. 2005). Peak performance of filter is captured while inducing in-plane angular rotations (ranging from $0^{\circ}$ to $360^{\circ}$ ) in the test image. 


\section{RESULTS AND DISCUSSION}

The example test image (Figure 3a) carries multiple distortions including in-plane and out-of-plane angular rotations, scale changes and synthetically embedded occlusion in the scene. The selected test object shown in Figure 3c portrays removal of clutter from the test image based upon matching of shape context information. As logarithmic versions of correlation filters are enabled to cater for multiple distortions, therefore distortion invariant technique like shape contexts provides us with an additional layer of validation. Apart from defiance of clutter/ non-target objects from the test image, we get enhanced results in correlation output leading to an improved classification decision.

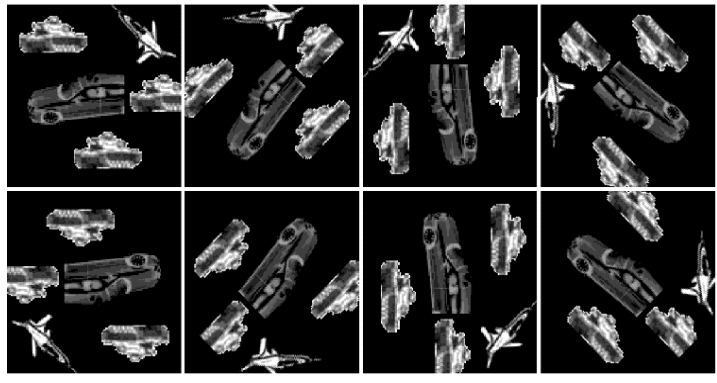

(a)

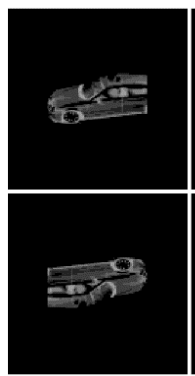

(c)
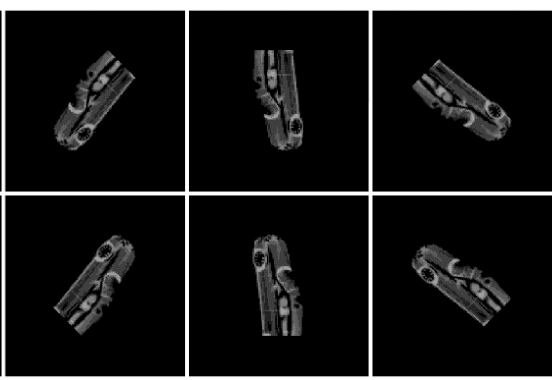
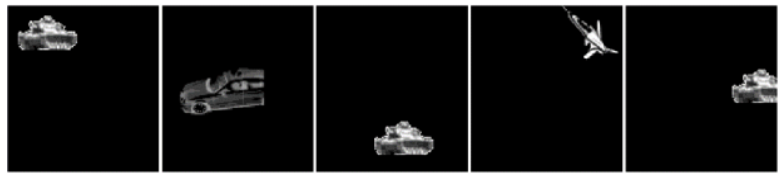

(b)

Figure 3. Example Test Image. (a) Rotated versions of Test-2 (0-360 degrees in-plane rotations). (b) Segregated Objects in Test-2. (c) Output Images (Reconstructed images after Clutter Removal)

Figure 4 displays a combined COPI difference plot against test images shown in Figure 2b. The COPI difference is calculated by subtracting the pre-selected threshold value from the correlation peak height and the difference is obtained in percentage form. A remarkable COPI difference percentage can be viewed in all 4 test cases, which is attributable to removal of clutter in the test image.

Clutter impacts on correlation output through occurrence of side lobes. These side-lobes in the correlation plane tend to distort output thus effecting classification decisions. The proposed treatment of clutter through shape context matching enables us to reduce the side-lobe contribution leading to a considerable improvement in peak to side-lobe ratio (PSR). PSR is a conventional benchmark parameter for ascertaining efficiency of the correlation filters.

PSR plots for all test images are displayed in Figure 4b. The prominent increase in PSR for all the test cases manifests efficacy of the proposed scheme. Percentage gain in peak to side-lobe ratio is also displayed against each angle for all 4 test images. Overall gain ranging from $30 \%$ to $70 \%$ can be viewed in peak to side lobe ratio in Table-1 below.

Table 1. \% Gain in Peak to Side Lobe Ratio for all test images in figure $2 \mathrm{~b}$

\begin{tabular}{|c|c|c|c|c|}
\hline \multirow{2}{*}{ In-Plane Rotation (Degrees) } & \multicolumn{4}{|c|}{ \% Gain Peak to Side Lobe ratio } \\
\cline { 2 - 5 } & Test Image-1 & Test Image-2 & Test Image-3 & Test Image-4 \\
\hline $5^{\circ}-30^{\circ}$ & $70.24 \%$ & $46.13 \%$ & $29.99 \%$ & $65.84 \%$ \\
\hline
\end{tabular}

\section{CONCLUSION}

An appropriate addition in the optimal LMACH design causes a considerable improvement in the results. The proposed filter SC-MACH effectively denies clutter thus making the classification decision free from any participation of clutter in the correlation output. This yields a performance gain ranging from $30 \%$ to $70 \%$ in 
the PSR, thus allowing correct classification of true class objects. The improvement remains consistent throughout the range of in-plane angular distortions against synthetically cluttered challenging images.

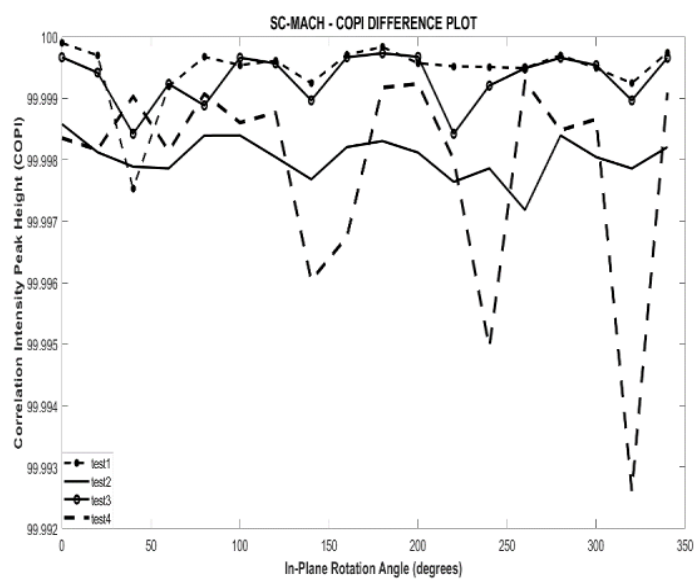

(a)
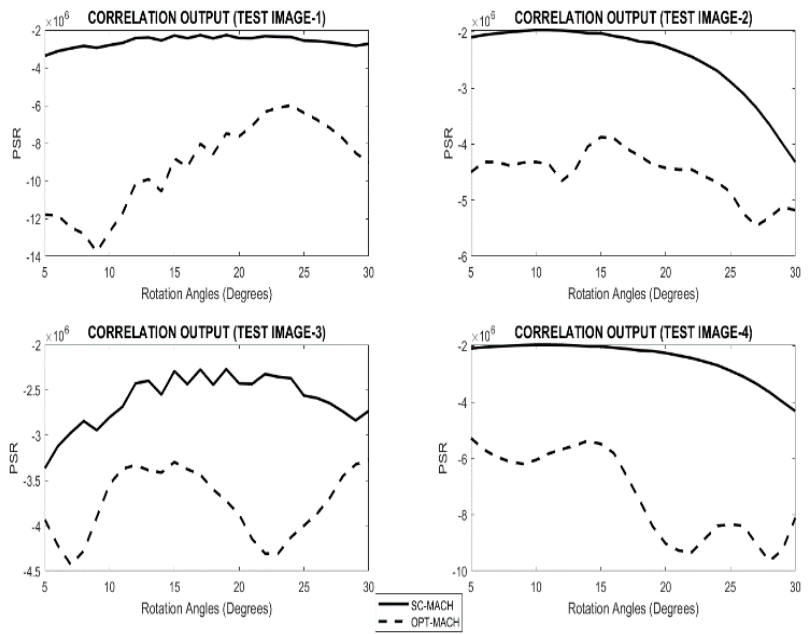

(b)

Figure 4. (a) COPI Difference Plot for SC-MACH Filter. (b) PSR Plots for Test Images, a PSR Plot test image-1, b PSR Plot test image-2, c PSR Plot test image-3, d PSR Plot test image-4

\section{REFERENCES}

Vijaya Kumar B.V.K., Mahalanobis A., Juday R.D., 2005. Correlation pattern recognition. Cambridge University Press, New York, USA.

Mahalanobis A., Vijaya Kumar B.V.K. and Song S., 1994. Unconstrained correlation filters. In Appl. Opt., 33, (17), pp. 3751-3759.

Alkanhal M., Vijaya Kumar B.V.K. and Mahalanobis A., 2000. Improving the false alarm capabilities of the maximum average correlation height correlation filter. In Opt. Eng., 39, (5), pp. 1133-1141.

Vijaya Kumar B.V.K. and Alkanhal M., 2001. Eigen-extended maximum average correlation height (EEMACH) filters for automatic target recognition. Proceedings of SPIE, Orlando, Florida, USA, pp. 424-431.

Hanying Zhou and Tien-Hsin Chao., 1999. MACH filter synthesizing for detecting targets in cluttered environment for grayscale optical correlator. Proceedings of SPIE - The International Society for Optical Engineering. 3715. $394-398$. $10.1117 / 12.341322$.

Bone P., Young R. and Chatwin C., 2006. Position, rotation, scale and orientation-invariant multiple object recognition from cluttered scenes. In Opt. Eng., 45, (7), p. 077203.

Ho C.G., Young R.C.D. and Chatwin C.R., 2002. Sensor geometry and sampling methods for space-variant image processing. In Pattern Anal. Appl., 5, (4), pp. 369-384.

Rehman S., Bilal A. and Javed Y., 2013. Logarithmically pre-processed EMACH filter for enhanced performance in target recognition. In Arab. J. Sci. Eng., 38, (11), pp. 3005-3017.

Rodriguez A., Boddeti V.N. and Vijaya Kumar B.V.K., 2013. Maximum margin correlation filter: a new approach for localization and classification. IEEE Trans. Image Process., 22, (2), pp. 631-643.

Fernandez J.A. and Vijaya Kumar B.V.K., 2015. Partial-aliasing correlation filters. IEEE Trans. Signal Process., 63, (4), pp. 921-934.

Tehsin S., Rehman S. and Bilal A., 2017. Comparative analysis of zero aliasing logarithmic mapped optimal trade-off correlation filter. Pattern Recognition and Tracking XXVIII, Anaheim, California, USA, p. 1020305.

Ahmed B. Awan, Saad Rehman and Asim D. Bakhshi, 2018. Composite filtering strategy for improving distortion invariance in object recognition. IET Image Processing, Online ISSN 1751-9667.

S. Belongie, J. Malik and J. Puzicha, 2002. Shape matching and object recognition using shape contexts. In IEEE Transactions on Pattern Analysis and Machine Intelligence, vol. 24, no. 4, pp. 509-522. 